\title{
SOME PROBLEMS WITH HTSC IN LOW-FREQUENCY ELECTRIC AND MAGNETIC MEASUREMENTS
}

\author{
S. MÉSZÁROS and K. VAD \\ Institute of Nuclear Research of the Hungarian Academy of Sciences, H-4001 Debrecen, P.O.B. 51., \\ Hungary \\ (Received January 23, 1993; in final form February 3, 1993)
}

\section{INTRODUCTION}

Sensitivity to magnetic fields is one of the electromagnetic properties of superconductors. Owing to some special effects, superconductors display extreme sensitivity to the presence, intensity, and frequency of electromagnetic fields. The discovery of tunnelling between two weakly coupled superconductors and especially the Josephson effects opened up new possibilities in the detection of extremely weak lowfrequency signals. Some of these new possibilities were exploited, but their proliferation has been limited by the need for using sophisticated cryogenic techniques to maintain the superconducting state. With the classical superconductors, the critical temperature $\left(\mathrm{T}_{\mathrm{c}}\right)$ was below about $24 \mathrm{~K}$, so the use of liquid helium or closed-cycle refrigerators producing temperatures below $20 \mathrm{~K}$ were unavoidable. This made the extended use of superconductive sensing elements more difficult. The discovery of high-temperature superconductors in 1986 offered a chance to eliminate the above limitation. However, specific properties cause problems in putting this advantage into practice. The cuprate family of high-temperature superconductors (HTSCs) have a layered structure and extremely short superconducting coherence length. As a consequence, no simple techniques are available to produce HTSC wires and films with high critical-current density and homogeneous structure. These requirements are important both in large-scale and smallscale applications. In this paper, we discuss the possibilities of using screen-printed HTSC films in superconducting magnetometers working at liquid nitrogen temperature. Thin-film deposition methods can produce higher quality films. However, the screen-printing technique, being much simpler and cheaper, is worthy of development efforts.

\subsection{Josephson Effects, SQUIDs and Their Use for Magnetometers}

Josephson effects appear when two superconductors are weakly coupled, i.e., when there is a connection between them enabling Cooper pairs to flow from one su- 
perconductor to the other. Josephson junctions can be constructed in several ways, including tunnel junctions, point contacts, and microbridges. A simple formalism using elementary quantum mechanics and perturbation theory can be used to derive the Josephson equations describing the general properties of Josephson junctions. These equations and their solutions corresponding to different situations are discussed in several books, review, and research papers, (see e.g. 1, 3, 2 and references therein). The basic Josephson equations are

$\mathrm{j}=\mathrm{j}_{0} \sin (\varphi)$

and

$V=(\hbar / 2 e) d \varphi / d t$

where $\varphi$ is the quantum phase difference across a selected point of the Josephson junction, $\mathrm{j}$ is the current density at the same point, and $\mathrm{j}_{0}$ is the maximum current density transferred by superconducting charge carriers, i.e., Cooper pairs. The quantum phase difference is connected to the magnetic vector potential $(\overrightarrow{\mathrm{A}})$ by the relation

$\varphi=2 \mathrm{e} / \hbar \int \overrightarrow{\mathrm{A}} \mathrm{d} \overrightarrow{\mathrm{s}}$

These equations, together with the Maxwell equations give a description of the electrodynamics of Josephson junctions. In the following, we limit our discussion to point-like Josephson junctions, i.e., we ignore the spatial changes of the quantum phase difference.

The simplest Josephson element is a small-sized junction connected to a current source and exposed to an electromagnetic field. The theoretical description is based on the RSJ (Resistively Shunted Junction) model $^{3}$ :

$\mathrm{C} \frac{\Phi_{0}}{2 \pi} \frac{\mathrm{d}^{2} \varphi}{\mathrm{dt}^{2}}+\frac{\Phi_{0}}{2 \pi \mathrm{R}} \frac{\mathrm{d} \varphi}{\mathrm{dt}}+\mathrm{I}_{\mathrm{c}} \sin \varphi=\mathrm{I}$

where $\mathrm{C}, \mathrm{R}, \mathrm{I}_{\mathrm{c}}$ are the junction capacitance, resistance, and critical current, $\Phi_{0}=$ $\mathrm{h} / 2 \mathrm{e}$ is the magnetic flux quantum, $\varphi$ is the quantum phase difference across the junction, and $I$ is the external drive current. The Josephson junction itself is sensitive to the magnetic field applied externally. The magnetic field causes the diffraction-like change in the critical current of the junction. the presence of a high-frequency electromagnetic field induces changes in the current-voltage characteristics of the junction. In this paper, we shall not discuss the use of single Josephson junctions as detectors. The static and low-frequency magnetic fields can also be detected by superconducting loops containing one or two Josephson junctions (RF or DC SQUIDs). The RF SQUID is a low inductance superconducting loop containing one Josephson junction. The readout of the state of the ring is performed by coupling it inductively to a radio frequency resonant circuit and monitoring its impedance. These instruments have been available commercially since the mid-70s. The DC SQUID is a low inductance superconducting loop 
containing two Josephson junctions. The macroscopic quantum interference is manifested in the critical current of the loop as a function of the magnetic flux introduced into it. The simplest technique to measure the response of the DC SQUID is to monitor the voltage across it when a DC current somewhat above the critical current is applied. Both RF and DC SQUIDs can be equipped with a suitable electronic feedback system in order to have a linear response and high dynamic range. The optimization of these instruments have been analyzed by several authors. The best noise performance was achieved by DC SQUIDs using fabricated tunnel junctions. The energy sensitivity of the best SQUIDs is close to the quantum limit ( $\hbar$ ) in the white noise region. This very impressive sensitivity, however, was obtained by SQUIDs having an inductance of $\sim 1 \mathrm{pH}$, so the coupling of external signals to the SQUID needs very careful design.

\subsection{High $T_{\mathrm{c}} S Q U I D s$ and Detectors}

Several experimentally published observations have proven that Josephson effects exist in HTSC materials. ${ }^{9}$ HTSC ceramics, screen-printed films, and even singlecrystals and thin films contain several weak couplings, many of them displaying Josephson junction features. The presence of these weak couplings between superconducting grains (called grain boundary junctions, GBJs) is responsible for many unfavorable properties of HTSC bulk materials, e.g., their low critical-current density $\left(j_{c}\right)$, rapid reduction of $j_{c}$ in a magnetic field, etc. These properties make the production of HTSC materials for large-scale applications like magnets, energy storage, and power transfer a challenging task. The applications in the measurement technique seem to be more feasible.

In order to have HTSC SQUIDs with characteristics comparable to their conventional counterparts, several technological problems are to be solved. The first requirements is to produce Josephson junctions with parameters suitable for RF or DC SQUIDs. This includes the preparation of tunnel junctions with low capacitance, size small compared to Josephson penetration depth, large $I_{c} R_{N}$ product, free of hysteresis, and with a relatively large critical current density. At the same time, somewhat surprisingly, high-quality SQUIDs were made using natural GBJs for Josephson junctions..$^{87}$

Another equally important requirement is the construction of high-quality transducers making SQUIDs capable of measuring different signals. These signals can be the magnetic field, voltage, current, displacement, or any other physical quantity. The transducer (e.g., a flux transformer in magnetometers) usually uses superconducting wire or film. The material the transducer in question is made of should have a high critical-current density and be free of magnetic hysteresis.

The role of natural grain boundaries is contradictory; they can serve as active Josephson junctions for SQUIDs making preparation of HTSC magnetometers easier on the one hand, but the construction of transducers used for coupling external signals to SQUIDs more difficult, on the other. To elucidate the nature of GBJs, we investigated the current transport properties of screen-printed HTSC films as a function of temperature, external magnetic field, and film width. The last parameter is very important because by changing the film width, we can change the number of GBJs in the current path. 


\section{EXPERIMENTAL DETAILS}

The samples used in our measurements were prepared by the method of Besenyei et al. ${ }^{4}$ They used the screen printing technique to produce superconducting films. As the first step, they prepared superconducting powders from an appropriate mixture of $\mathrm{Bi}_{2} \mathrm{O}_{3}, \mathrm{SrCO}_{3}, \mathrm{CaCO}_{3}, \mathrm{CuO}$ and $\mathrm{PbO}$ in order to give the nominal composition of the 2223 phase as $\mathrm{Bi}_{1.96} \mathrm{~Pb}_{0.34} \mathrm{Sr}_{2.03} \mathrm{Ca}_{1.91} \mathrm{Cu}_{3.06} \mathrm{O}_{\mathrm{x}}$. (For control measurements the 2212 phase was also prepared.) The powders were mixed in an agate mortar and calcined at $780^{\circ} \mathrm{C}$ for 5 hours in the air. After they were pulverized, they were heat treated at $780^{\circ} \mathrm{C}$ for 25 hours. A paste was made from the milled powder by thoroughly mixing it with an organic binder and some solvents. As the second step, thick films were prepared on $96 \%$ polycrystalline $\mathrm{Al}_{2} \mathrm{O}_{3}$ substrates by the screen printing technique. Finally, it was fired in a conveyor furnace with the usual temperature-time profile, involving $10 \mathrm{~min}$ at top temperature and $50^{\circ} \mathrm{C} / \mathrm{min}$ heating and cooling rate. The microstructure of the films was studied by scanning electron microscopy and X-ray diffraction measurements.

Current and voltage contacts to the films were provided with burnt-in silver paste pads with a contact resistance of less than $50 \mathrm{mohm}$. The average thickness of the films was $30 \mu \mathrm{m}$, their original width $1 \mathrm{~mm}$, and the distance between the potential contacts $6 \mathrm{~mm}$. The film width was systematically reduced by mechanical scraping of the film between the potential contacts. As this process may cause the remaining film to crack, the state of the remaining film was checked by measuring its normal state resistivity.

Current-voltage $(\mathrm{I}-\mathrm{V})$ characteristics were recorded by continuous and pulsed current methods with the conventional four-probe arrangement. The temperature and external static magnetic field dependence was studied by placing the sample into a temperature-controlled chamber inside the bore of a $6 \mathrm{~T}$ superconducting magnet. The temperature ranging from $4.5 \mathrm{~K}$ to $150 \mathrm{~K}$ was controlled by a $\mathrm{He}$ gas stream. ${ }^{5}$ If the width of the film is less than $\sim 0.5 \mathrm{~mm}$, the current may cause permanent damage to it. This was prevented by connecting a shunt resistor across the potential contacts and so the true I - V characteristics were calculated.

In order to see macroscopic quantum interference, the voltage was measured as a function of external magnetic field at fixed DC current. Because very small field change was necessary in this experiment, a small coil was fastened to the sample holder with its axis perpendicular to the film surface and was driven by a low-level current generator. In order to exclude external magnetic noises, the measurement was done in a superconducting shield (lead tube). The stability of the output voltage was studied by recording it as a function of time while the transport current and magnetic filed were kept constant.

The quality of the superconducting films used in our investigations was checked by measuring the resistance-temperature $(\mathrm{R}-\mathrm{T})$ characteristics in different magnetic fields and the critical currents at different temperatures. $R-T$ curves of 2223 and 2212 phases are presented in Fig 1 . It can be seen that the pure 2223 phase could not be synthesized, since the 2212 phase was also present in the 2223 phase. In Figs 2 and 3, the current-voltage characteristics measured in different 


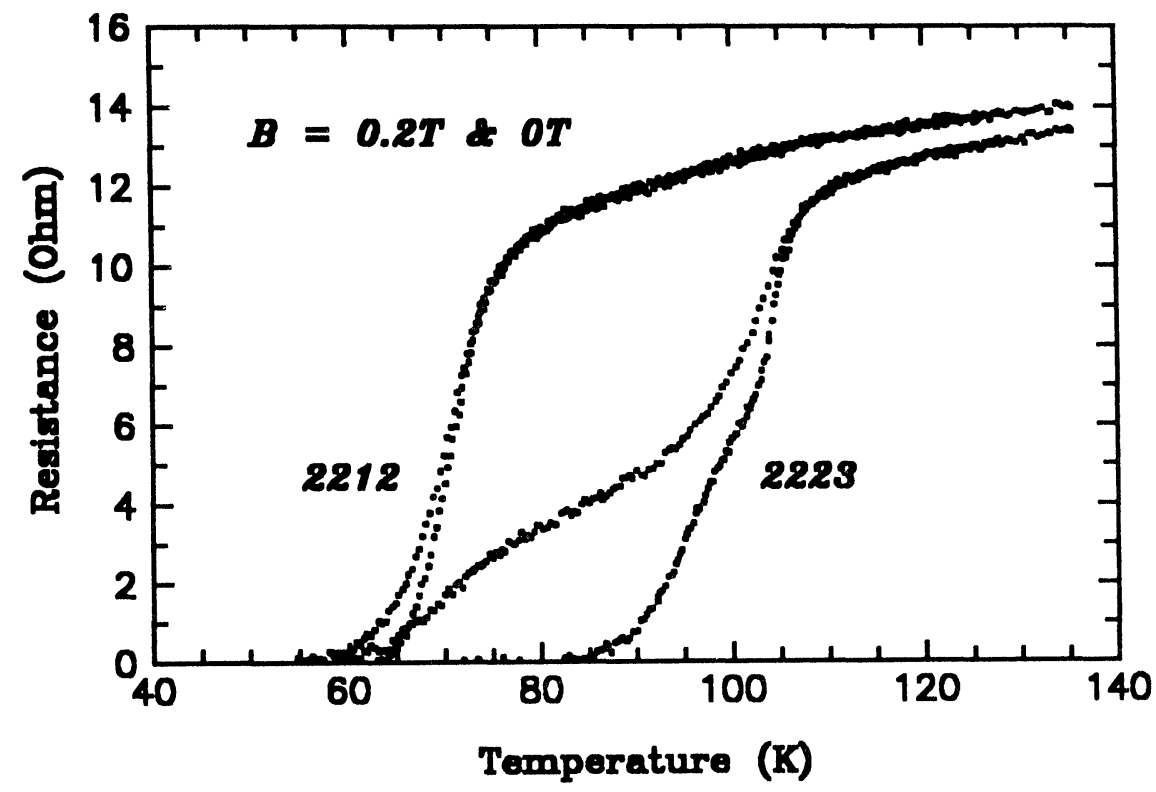

FIGURE 1 Resistance-temperature characteristics of the two types of samples used in our measurements. The 2212 sample is a single phase one while the 2223 is mixed.

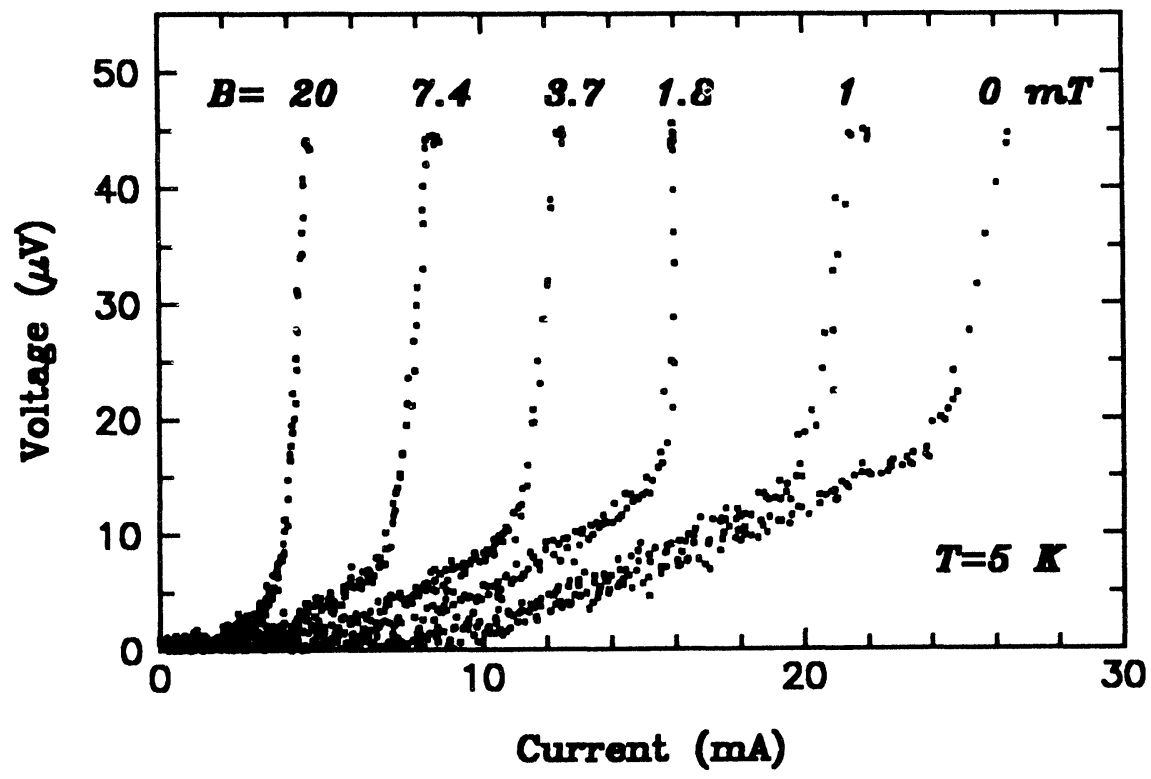

FIGURE 2 Current-voltage characteristics in different magnetic fields. The sample width is $1 \mathrm{~mm}$. 


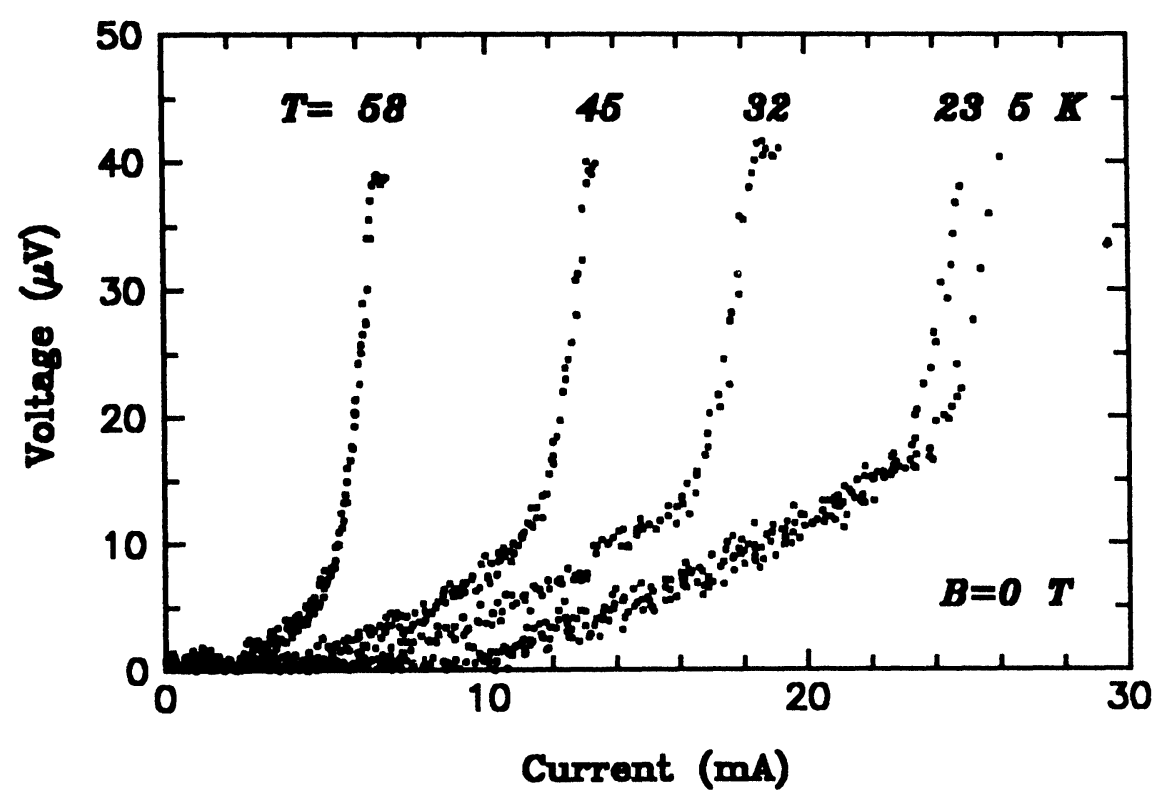

FIGURE 3 Temperature dependence of I - V curves.

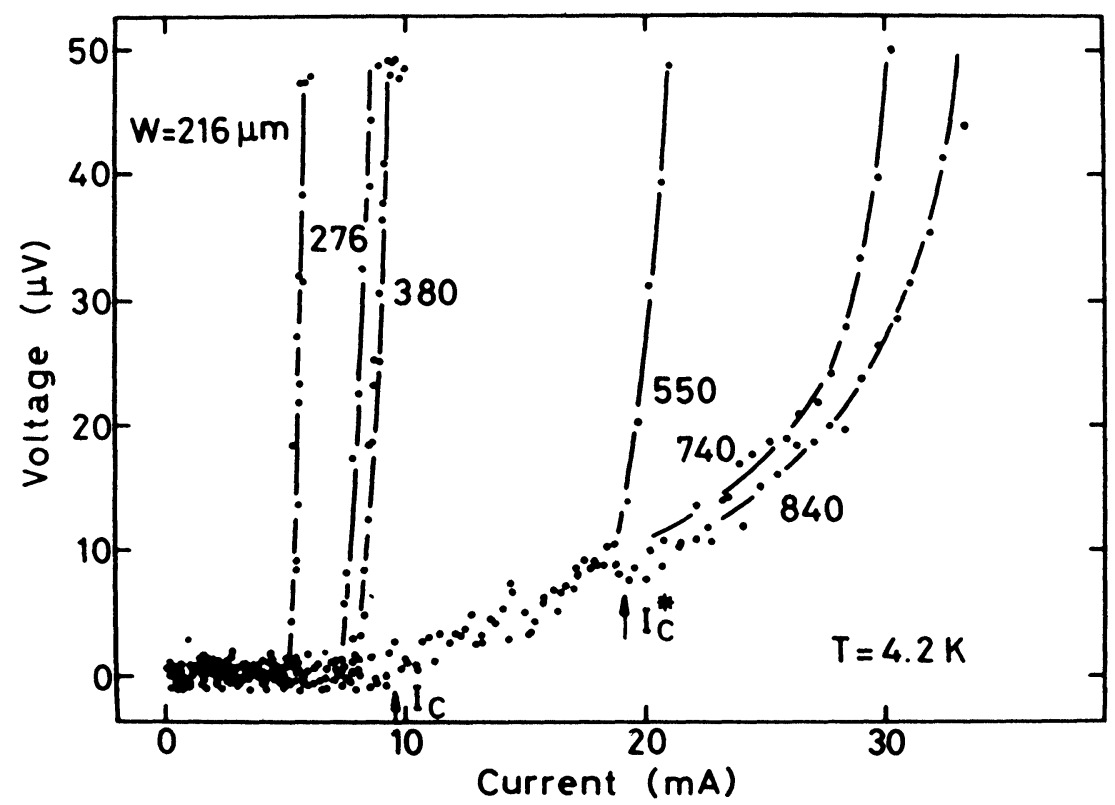

FIGURE 4 I - V curves as a function of film width in zero magnetic field at $4.2 \mathrm{~K}$. 
magnetic fields and at different temperatures can be seen. Their film width dependence is shown in Fig 4.

Both $\mathrm{R}-\mathrm{T}$ and $\mathrm{I}-\mathrm{V}$ curves were measured in all the three relative directions of the film normal vector and the magnetic field. The results we found were independent of the direction of the magnetic field, i.e., apart from the differences in the demagnetization factor the curves were independent of the Lorentz force.

\section{RESULTS AND DISCUSSION}

Fig 4 shows the size dependence of I - V curves. At larger film widths the voltage appears at a current value independent of the film width and all the curves follow the same line up to a turning point, where a steep increase of the voltage takes place. At smaller film widths, the steep increase starts directly from the zero voltage state. This feature appears at higher temperatures and in magnetic fields too. It is important to note that quantum interference is observed only above the turning point. When the critical current was defined as the position of the turning point, a good correlation was found with the normal state resistance ${ }^{6}$ (see Fig 5.). This means the the film can transfer supercurrent, but the presence of GBJs prevents

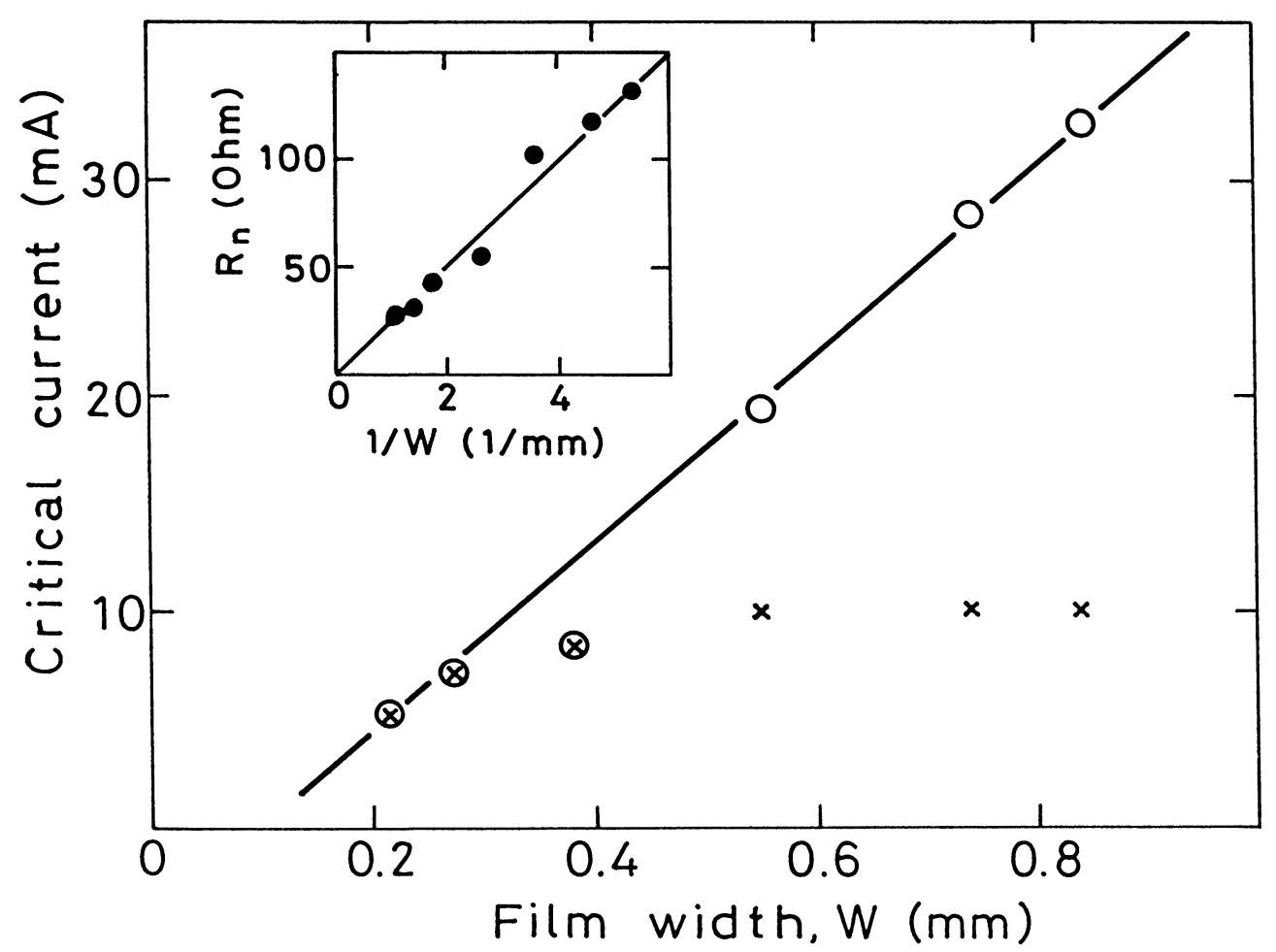

FIGURE 5 Film width dependence of critical current determined from: $x 3 \mu \mathrm{V} / \mathrm{cm}$ criterion; o turning point position. Inset shows the width dependence of normal state resistance. 
us from increasing the current by enlarging the cross section of the film. A detailed study of this mechanism is under way in our lab. Above the turning point, GBJs give a well-defined quantum interference as it can be seen in Fig 6 . The lower trace is the output voltage as a function of magnetic field displayed on the lowermost trace. This character is again universal in the sense that it appears at temperatures up to $T_{c}$ and magnetic fields up to $1 \mathrm{~T}$. The features of this pattern resemble a multi-junction DC SQUID. The amplitude of the pattern is high enough for measurement purposes. However, we faced the problem of stability of the interference pattern. When the magnetic field change was stopped and the output voltage was recorded, the curves shown on the upper two traces in Fig 6. were obtained. The two curves differ in the time scale. The voltage is not stable despite the fact that the measurement was performed in a superconducting shield excluding external magnetic disturbances. (This was checked by putting a conventional SQUID magnetometer in the same shield.) The voltage change resembles a chaotic motion of

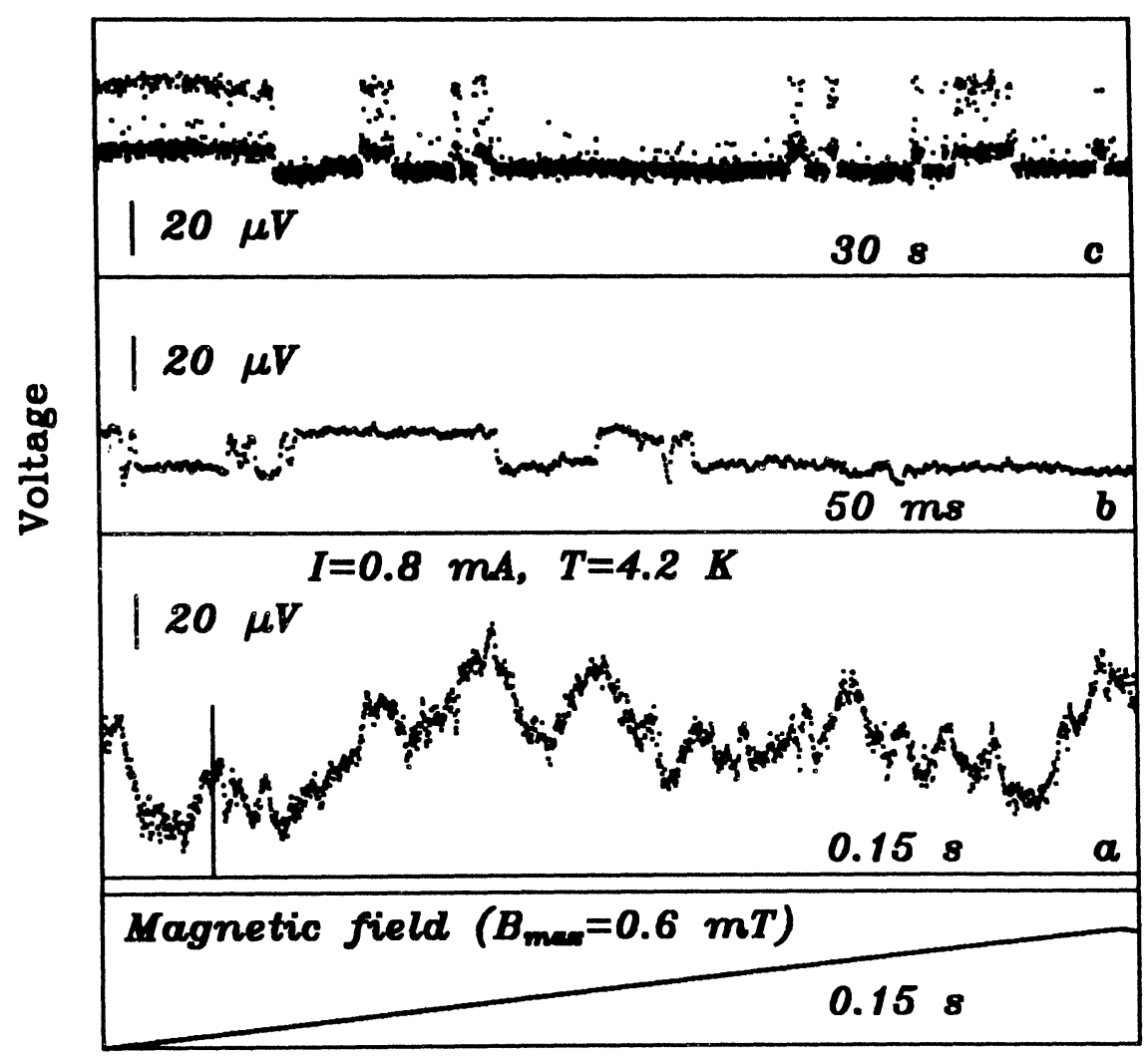

\section{Time}

FIGURE 6 Quantum interference patterns in a screen printed film. $a$ : output voltage as a function of external field at fixed current $b$ and $c$ : output voltage at fixed current and magnetic field lowermost trace: change of the magnetic field in time. 
magnetic flux in the GBJs. This interpretation is supported by the facts that the phenomenon was found to be independent of the temperature and that the amplitude distribution of the voltage had defined peaks.

In conclusion we can say that the nature of GBJs is technology dependent, and the elimination of weak GBJs from the transducers is very important, especially if the transducer is expected to carry high currents. GBJs in screen-printed films seem to be unsuitable for patterned DC SQUIDs. The importance of preparation of screen-printed films with high critical-current density GBJs is strongly recommended.

\section{ACKNOWLEDGEMENT}

This work was supported by the Hungarian Scientific Foundation (OTKA) under contract No. T-4049.

\section{REFERENCES}

1. L. Solymar, Superconductive Tunnelling and Applications, Chapman and Hall Ltd, London, 1972.

2. J. C. Gallop, SQUIDs, the Josephson Effects and Superconducting Electronics, Adam Hilger, 1991.

3. A. Barone and G. Paternò, Physics and Application of the Josephson Effect, John Wiley \& Sons, 1982.

4. E. Besenyei, G. Katona, P. Arató and A. Kele, Supercond. Sci. Technol. 2, (1989) 220.

5. S. Mészáros, G. Halász, K. Vad and N. Hegman, Proc 6th Int. Symp. on Weak Superconductivity, eds. B̌enačka, M. Darula and M. Kedro, World Scientific, 1991, p. 56.

6. S. Mészáros, K. Vad, G. Halász, N. Hegman and G. Katona, Supercond. Sci. Technol., 4, (1991) S367.

7. B. Vasiliev, A. G. Lihachev, V. N. Polushkin, S. V. Uchaikin, Preprint of the Joint Institute for Nuclear Research, Dubna, Russia, N. D13-89-810 (in Russian).

8. S. Harrop, C. M. Muirhead, M. S. Colclough and C. E. Gough, Physica C 153-155 (1988) 1411.

9. G. B. Donaldson, M. Odehnal, C. M. Pegrum and J. R. Buckley, Physica C 153-155 (1988) 1407. 

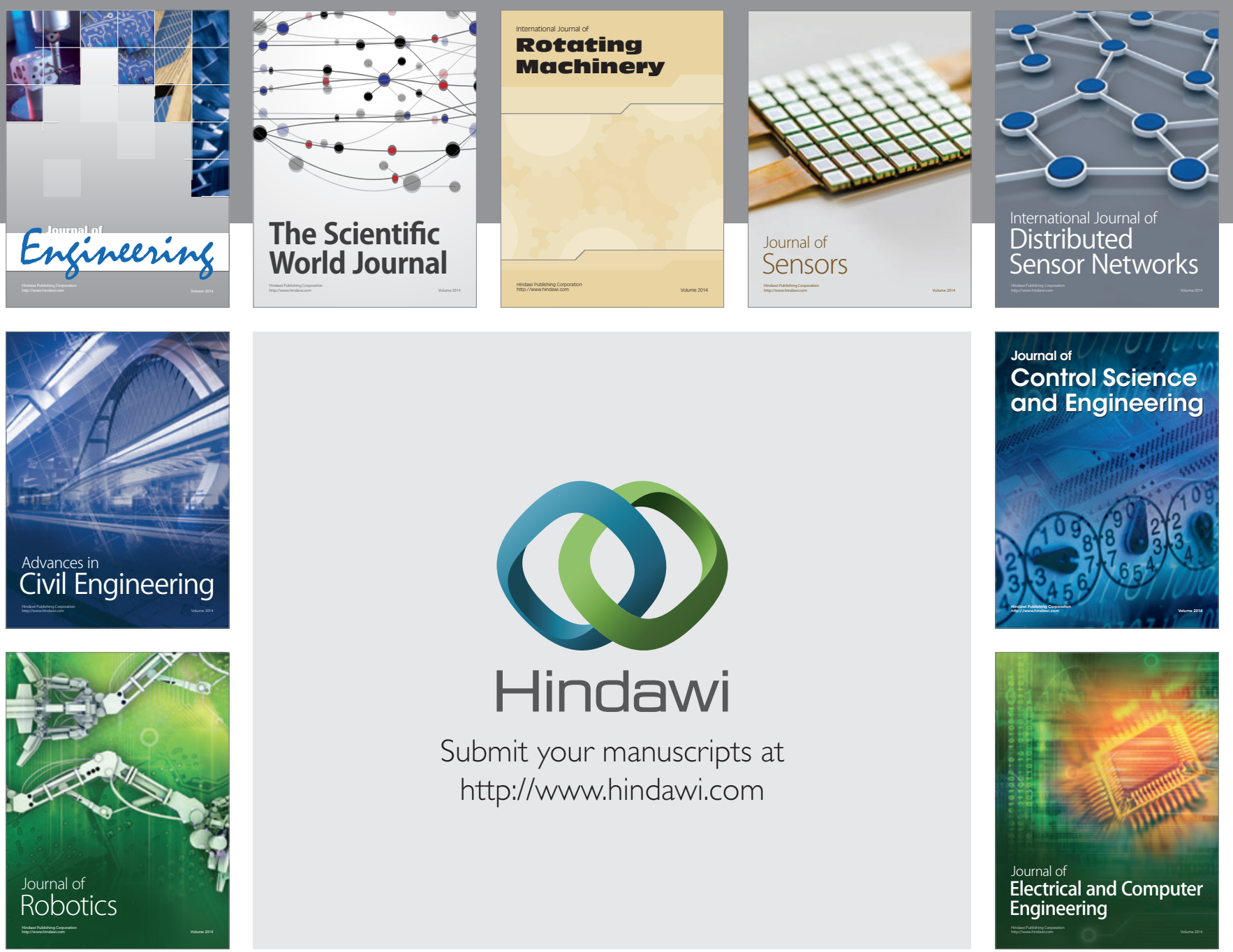

Submit your manuscripts at

http://www.hindawi.com
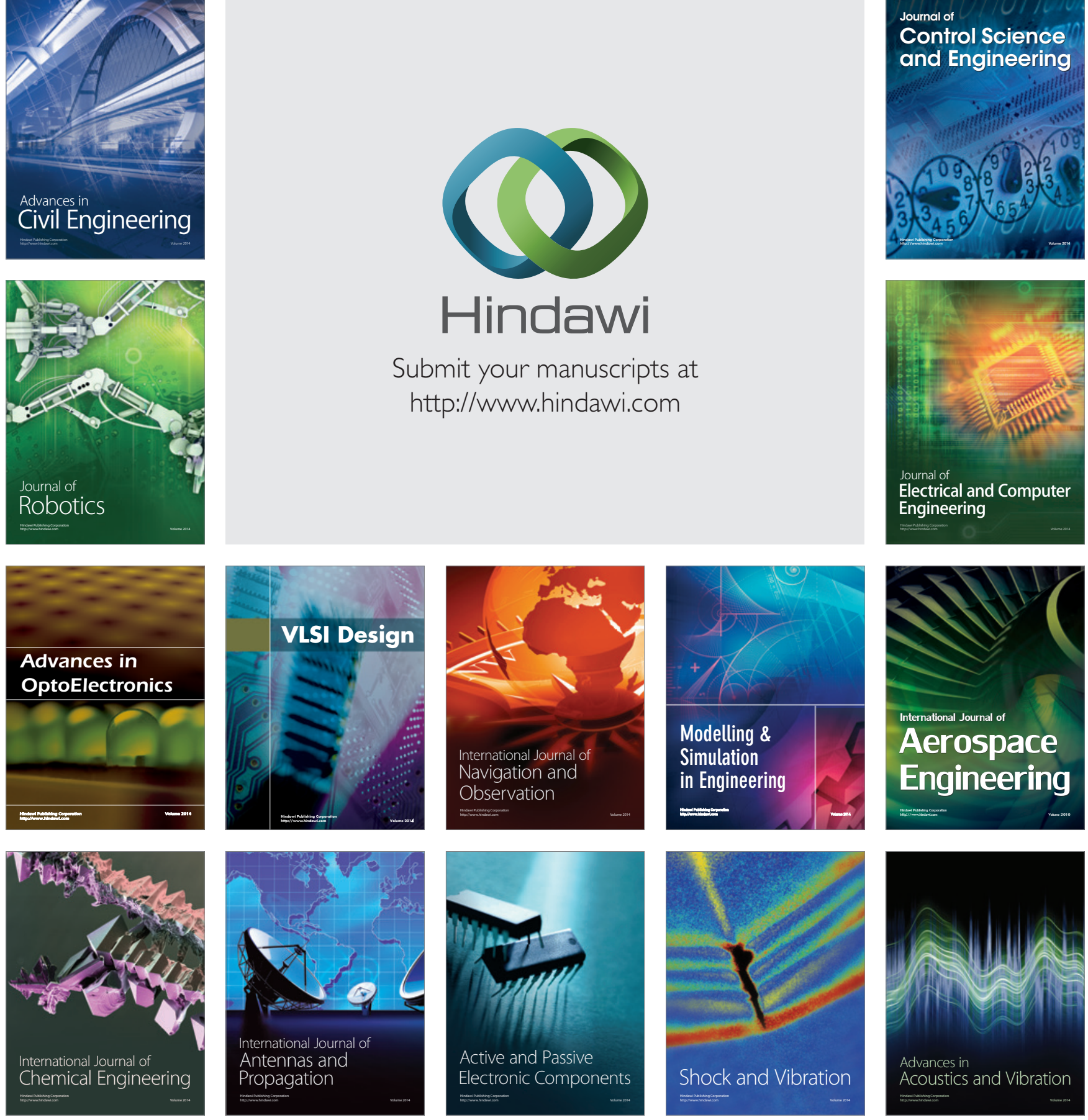